\title{
Biodiversidade: o enfoque interdisciplinar brasileiro
}

\author{
Biodiversity: the brazilian interdisciplinary \\ approach
}

Otto R. Gottlieb 1

Maria Renata de M. B. Borin 1

Carmen Lucia A. da C. Pagotto 1

Dorothea H. T. Zocher 1

1 Departamento de Fisiologia e Farmacodinâmica, Instituto Oswaldo Cruz, Fiocruz, Rio de Janeiro, RJ, 21045-900, Brasil.
Abstract In Brazil biodiversity, specially plant diversity, is considered an important source of wealth. Clearly, however, any enduring retribution in social benefits does not depend simply on the use of biodiversity, but on its sustainable use. Regional descriptions of fauna and flora constitute a first step in this direction. Utilized for mapping and quantification of biodiversity, such descriptions allow mechanistic interpretations of the functioning of nature. Without the understanding of these mechanisms "sustainable development" remains what it is today: a political appeal without social retribution (Beardsley, 1994). Our efforts aim to provide a secure theoretical basis for the elucidation of this "most marvellous mystery of life, the means by which so much diversity was generated from so little physical material" (Wilson, 1992). After all, "how can you carry out a national poli$c y$ - or even make intelligent decisions on your own life - if you do not understand the underlying questions" (Sagan, 1996)? This is a genuine Brazilian problem requiring an urgent gathering of efforts, since it has direct practical applications on the socio-economical well-being and the health of the people.

Key words Biodiversity; Quantitative Chemical Biology; Ecology; Systematics; Evolution; Natural Products
Resumo No Brasil, biodiversidade - em especial, a diversidade vegetal - é considerada importante fonte de riqueza. Inequivocamente, porém, qualquer retribuição durável em benefícios sociais dependerá não do uso da biodiversidade, mas sim de seu uso sustentável. Descrições regionais da fauna e da flora constituem um primeiro passo nessa direção. Quando utilizadas para mapeamento e quantificação da biodiversidade, permitem interpretar os mecanismos de funcionamento da natureza. Sem a compreensão desses mecanismos o "desenvolvimento sustentado" permanecerá o que é hoje: apelo político sem retribuição social (Beardsley, 1994). Nossos trabalhos pretendem fornecer segura base teórica para esclarecer esse "mais maravilhoso mistério da vida, o meio pelo qual tanta diversidade surgiu de tão pouca matéria física" (Wilson, 1992). Afinal, "como podemos executar a política nacional - ou até mesmo tomar decisões inteligentes sobre nossas próprias vidas - se não compreendemos as questões subjacentes" (Sagan, 1996)? Trata-se de problema autenticamente brasileiro que necessita da urgente unificação de esforços, porque de sua solução dependem aplicações práticas diretas para garantia do bem-estar socioeconômico e da saúde do povo.

Palavras-chave Biodiversidade; Químico-Biologia Quantitativa; Ecologia; Sistemática; Evolução; Produtos Naturais 


\section{Introdução}

Biodiversidade hoje no Brasil - uma das últimas fronteiras biológicas do mundo - constitui importante fonte de riqueza. No entanto, seu impacto para a garantia do bem-estar e da saúde do povo somente será atingido por meio do conhecimento das interações recíprocas dos organismos. Essa meta exige o discernimento da linguagem da natureza a fim de que se possa entender, participar e interferir no seu funcionamento. Que não haja dúvida a respeito: a maior parte dessa forma de comunicação é constituída por vocábulos químicos.

Infelizmente, o uso pleno da linguagem para a compreensão dos processos da vida é impedido pelo abismo que separa Química de Biologia. Estas áreas do saber conformam culturas distintas entre si, e o fato de permanecerem isoladas acarreta sérios problemas, é desconsiderado e contraprodutivo (Kornberg, 1987). Em conseqüência, um novo tipo de sistemática - ciência que estuda a diversidade dos organismos (Mayr, 1991) - precisa unir a Química a todos os níveis de organização dos seres vivos, com a esperança de que se venha a adquirir visão mais consistente da vida inserida em determinado ambiente.

Para isso, não basta utilizar uma abordagem multidisciplinar. É óbvio que simples descrições regionais da fauna e da flora, embora de interesse primordial, nunca levarão à compreensão do funcionamento da natureza, condição sem a qual o "desenvolvimento sustentado" permanecerá o que é hoje: apelo político sem retribuição social. Como podemos executar a política nacional - ou até mesmo tomar decisões inteligentes sobre nossas próprias vidas - se não compreendemos as questões subjacentes? (Sagan, 1996)

De fato, a biodiversidade, no estágio descritivo em que se encontra atualmente, não passa de assunto para reuniões técnico-científicas, uma vez que não consegue responder três perguntas fundamentais, pré-requisitos para qualquer aplicação prática do conceito. Biodiversidade: Onde ela está? Por que lá se encontra? O que acontecerá com ela? As explicações exigem uma abordagem interdisciplinar que ainda carece de metodologia consensual. Somente dessa maneira será possível desenvolver base teórica segura, capaz de avaliar e medir biodiversidade (Gottlieb \& Borin, 1994a).
Com essa finalidade, nosso grupo propôs uma metodologia original, que tem por base o mapeamento e a quantificação de dados químicos e biológicos (Gottlieb \& Borin, 1994a; 1996; Gottlieb et al., 1996a). Os dados químicos essenciais firmam-se em levantamentos realizados nas principais obras de referência, como Chemical Abstracts e Dictionary of $\mathrm{Na}$ tural Products (Buckingham, 1994-1997). Os dados biológicos fundamentais encontram apoio em inventários florísticos (Gottlieb \& Borin, 1996), etnobotânicos e em farmacopéias, assim como em arrolamentos de espécimens depositados nos principais herbários brasileiros (Bosisio et al., 1998).

Essa metodologia introduz a fitoquímica quantitativa como poderosa ferramenta inovadora. Aplicado ao estudo da evolução, o método dá lugar à racionalização de fenômenos ambientais em direção a uma ecologia mais exata mediante a integração de metabolismo, morfologia e ecogeografia, da mesma forma que possibilita o mapeamento e a quantificação da biodiversidade. A aplicação da fitoquímica quantitativa permitiu alcançar várias conclusões, todas demonstrando que mecanismos metabólicos são análogos a mecanismos biológicos nos níveis celulares, morfológicos e ecogeográficos (Gottlieb \& Borin, 1998a). Os exemplos escolhidos referem-se à substituição (ecologia), separação (sistemática) e seleção (evolução).

\section{Ecologia}

1) Quantificação da biodiversidade envolve em geral apenas a determinação do número de espécies em uma área. Acredita-se que esse método demonstre a diminuição de diversidade de espécies desde os trópicos até os pólos, concepção tida como "verdadeiro princípio geral da biologia" e que foi racionalizada por meio do decréscimo concomitante em energia solar (Wilson, 1992). Em vista do significado limitado desse tipo de quantificação, introduzimos novo indicador, denominado Singularidade Taxonômica (ST) (Gottlieb \& Borin, 1994a; 1996; Gottlieb et al., 1996a), que mede distâncias morfológicas médias por comparação par a par de todas as espécies registradas para a área. A determinação do ST e de outros parâmetros evolutivos - o Índice de Sporne (1980) e o Índice de Herbacidade (Borin \& Gottlieb, 1993; Gottlieb, 1982; Gottlieb 
et al., 1996a) para inventários florísticos sulamericanos - demonstrou, ao contrário da expectativa, aumento gradual de tais índices com a distância crescente do Equador (Borin \& Gottlieb, 1994a; 1997; Gottlieb et al., 1995a; 1996a).

2) Com o objetivo de estudar e comparar as tendências da variabilidade micromolecular da flora angiospérmica, seria necessário o estudo fitoquímico de todas as espécies de cada região. Uma vez que essa tarefa é impraticável, desenvolvemos o conceito de perfis metabólicos universais. A metodologia consiste em inferir a composição micromolecular em determinada família angiospérmica por intermédio dos dados registrados na literatura para todas as suas espécies quimicamente conhecidas (Gottlieb \& Borin, 1994a; 1997a, b; Gottlieb et al., 1996a). A construção de mapas fitoquímicos para áreas sul-americanas mostrou três padrões: a) Predominância de produtos naturais derivados de ácido chiquímico - tais como neolignanas - em espécies florestais de baixas latitudes. b) Predominância de produtos naturais derivados de ácido acético - tais como poliacetilenos - em espécies de cerrado de altas latitudes. c) Variações de perfis metabólicos totais bem mais fracas em florestas do que em cerrados (Gottlieb \& Borin, 1994a; 1997a, b; Gottlieb et al., 1996a).

3) Utilizando como base experimental inventários florísticos de áreas sul-americanas, verificamos que as mudanças morfológicas e químicas da flora angiospérmica que acompanham as passagens de um bioma para outro não acontecem como substituições latitudinais graduais - tão apregoadas pela literatura -, mas ocorrem via ecotonos, que são repentinas transições geográficas com faixas limítrofes turbulentas, semelhantes às transições de fase físico-químicas. Nessas zonas ecogeográficas de tensão caracterizadas pela quebra dos gradientes morfo-químicos surge maior complexidade estrutural e molecular (Gottlieb \& Borin, 1994a; 1997a, b), por razões que estamos investigando.

4) A família Rubiaceae, em virtude de ser uma das maiores famílias vegetais e encontrarse distribuída notadamente em regiões neotropicais, serviu como excelente base experimental para o estudo da diferenciação morfoquímica em radiações latitudinais. A integração entre classificação botânica, perfil metabólico e distribuição ecogeográfica de suas espécies sul-americanas levou ao reconhecimen- to e ao mapeamento de ecotonos. As regiões fronteiriças entre ecossistemas contrastantes - como, por exemplo, floresta Amazônica/cerrado, restinga/savana e planalto central/pampas - favorecem a adaptação de Guettardeae - a mais importante tribo neotropical da subfamília Antirrheoideae - e sofrem maior diversificação morfológica e metabólica por espécie do que os vastos ecossistemas vizinhos (Gottlieb \& Borin, 1996).

Adicionalmente, outras famílias foram utilizadas para determinar efeitos morfológicos e químicos da radiação espacial longitudinal de vegetais (Gottlieb, 1982; Pagotto et al., 1998). A sistematização dos padrões e princípios fitogeográficos observados servem para a criação de um modelo de diferenciação morfo-química de linhagens vegetais.

5) O estudo ecogeográfico de Rubiaceae abriu vistas para um fenômeno de natureza geral. A semelhança entre padrões morfo e químico-geográficos sugeriu a existência, na família, da tendência geral - previamente observada para angiospermas (Gottlieb, 1990) - de deslignificação e eliminação de metabolitos secundários derivados de chiquimato, possivelmente induzidas via canalização evolutiva. Na subfamília mais recente, Rubioideae, essa propensão pode ser atenuada por lignificação secundária, provavelmente induzida por mecanismo de retroalimentação que envolve ácido gálico (Gottlieb et al., 1995b). As transições dinâmicas entre formas diferentemente lignificadas constituem indicadores da proporção de metabolitos derivados de chiquimato e de acetato ou, em outras palavras, são indicadores do poder relativo de atração metabólica pelos caminhos do chiquimato e do acetato, "um verdadeiro princípio geral da Biologia”.

6) Em gêneros de Lauraceae, substituições evolutivas de lactonas sesquiterpênicas - primeiro por benzilisoquinolinas e neolignanas e, finalmente, por pironas - acompanham transições geográficas da Ásia (via uma rota equatorial) ou da Austrália (via uma rota antártica) em direção a América. A modulação de feições metabólicas em moldura dinâmica permite a clarificação de problemas referentes à sistemática de gêneros de Lauraceae ( $\mathrm{Pa}$ gotto et al., 1998).

7) A Amazônia e os restantes ecossistemas neotropicais, por um lado, sustentam uma rede integrada de plantas floríferas, na qual ecotonos funcionam como transmissores de tá- 
xons e de informação. A descoberta dessa rede vegetal tem conseqüências potenciais tão profundas para o continente que urge investigar suas causas e esclarecer os possíveis efeitos. Por outro lado, as zonas heterogêneas de transição - não os ecossistemas mais homogêneos - comumente representam a fonte básica de desenvolvimento futuro (Gottlieb \& Borin, 1998b). O seu estudo é de importância central. Vale a pena lembrar que, enquanto ecotonos naturais são selecionados para o assentamento de famílias de imigrantes na Amazônia, ecotonos artificiais são gerados para preservar corredores entre ecossistemas, base da estratégia moderna de conservação.

8) A quantificação dos teores lignínicos da vegetação no Fanerozóico permite, por extrapolação, prever a tendência do hábito vegetal, providenciando mesmo uma medida de status evolutivo. Torna-se possível, pela primeira vez, deduzir o teor de oxigênio da atmosfera em tempos passados não só levando em conta substâncias carbônicas diagenéticas em sedimentos, mas por intermédio da consideração de plantas fossilizadas em camadas geológicas (Gottlieb et al., 1995a).

Com base em novos conceitos teóricos sobre o funcionamento da natureza, esses trabalhos levam a uma série de possíveis aplicações práticas, como, por exemplo: mapeamento da composição química de plantas, fundamento para a busca das substâncias bioativas; avaliação quantitativa do potencial químico de uma área, sustentáculo científico para propostas de zoneamento; quantificação da lignificação angiospérmica, medida da biodiversidade; quantificação do conteúdo lignínico da vegetação no Fanerozóico, base de previsões da tendência do hábito de plantas; quantificação da biodiversidade, base de dados essenciais para o planejamento do uso sustentável de recursos naturais.

\section{Sistemática}

9) Tendências evolutivas em direção à biodiversidade são percebidas pelo exame quantitativo de gradientes metabólicos, tais como, em angiospermas, o gradual encurtamento do caminho biossintético do chiquimato via canalização evolutiva e o incremento gradativo do nível de oxidação médio das substâncias componentes de categoria metabólica particular. Essas transições metabólicas coincidem, em primeira aproximação, com a separação dos blocos magnolialeano e rosifloreano. Processos oxidativos de biossíntese simples seja pelo caminho do chiquimato seja pelo do acetato caracterizam respectivamente as subclasses Magnoliidae e Asteridae, ao passo que caminhos mistos não oxidativos distinguem uniformemente o complexo Hamamelidade, Dilleniidae, Rosidae (Gottlieb \& Borin, 1998c).

10) Se plantas floríferas são de fato monofiléticas, as categorias micromoleculares excepcionais e diferenciadas da subclasse Dilleniidae podem ser atribuídas à repentina introdução evolutiva, seguida pela gradual eliminação de polifenóis derivados de ácido gálico (Zocher et al., 1998).

11) Análise dos inventários publicados das plantas floríferas utilizadas para fins medicinais e alimentícios por três tribos de índios da Amazônia, Chácobo, Kayapó e Ka’apor, indicaram que, embora essas populações habitem regiões com vegetação completamente diferente, existe preferência comum às três sociedades por espécies vegetais mais evoluídas para propósitos medicinais, tal como por espécies mais primitivas para fins alimentícios (Gottlieb \& Borin, 1997c; Gottlieb et al., 1995c).

12) Resultados semelhantes foram obtidos no estudo dos hábitos alimentares de três espécies de macacos da Amazônia. De modo análogo aos humanos, macacos demonstram preferir plantas mais primitivas e, portanto, mais lenhosas, para sua alimentação. Os poucos dados publicados sobre o uso de plantas com fins medicinais por macacos, quando analisados segundo nossa metodologia, também mostram as mesmas preferências encontradas para as populações indígenas (Gottlieb et al., 1996b).

\section{Evolução}

13) Darwin desconhecia o conceito de autopoiese (auto-organização) molecular. Em sua época de atuação, metabolismo era entidade críptica, escondida em uma caixa preta, a célula. O conceito da seleção natural é, não obstante, tão esclarecedor no tocante à racionalização do fenômeno da biodiversidade que continua merecendo discussão construtiva (Gottlieb et al., 1996a). Deve-se isto ao fato de as observações de Darwin, apesar de restritas a organismos, terem revelado analogias moleculares com respeito à substituição, separação e seleção (Gottlieb \& Borin, 1998a). 
14) As controvérsias no que tange ao mecanismo evolutivo das plantas floríferas permanecem apesar das tendências atuais em favor da análise de dados macromoleculares e do crescente volume de dados micromoleculares. A abordagem narrativa usualmente empregada em quimiossistemática foi por nós substituída por técnica inovadora, a fitoquímica quantitativa. Sua aplicação revela a modulação de antagonismos como mecanismo fundamental de ecologia evolutiva em angiospermas. Origem e operação de muitos sistemas podem ser racionalizados de modo análogo. Conclui-se que o impacto de feições opostas possui relevância universal (Gottlieb \& Borin, 1998d).

15) O desenvolvimento evolutivo criador de sistemas abióticos e bióticos requer a alternância de forças agonísticas e antagonistas (ações e freios). Transições evolutivas operam, por exemplo, no universo (expansão e contração), no sol (massa e duração), na química pré-biótica (reprodutibilidade e estabilidade moleculares, macromoléculas e micromoléculas), na bioquímica (ciclo de Calvin e ciclo de Krebs, polifenóis e micromoléculas, via do chiquimato e via do acetato, galato e cafeato), na biologia (biodiversidade e bioparsimoniosidade, lenhosidade e herbacidade, ativação e desativação de sistemas celulares) e na ecologia (ecossistemas e ecotonos). A abrangência dos casos sugere tratar-se de mecanismo universal obedecido, segundo ritmos particulares, tanto pelo gradualismo filético (proposto por Darwin) como pelo equilíbrio pontuado preconizado por Eldredge e Gould (Eldredge, 1995). A extrapolação de tais evidências sugere que o significado da vida humana também deva ser controlado por antagonismos (Gottlieb \& Borin, 1998e).

\section{Conclusão}

A Ciência amanhã terá que estar preparada para fornecer respostas a questões ligadas aos mecanismos do funcionamento da natureza. "À medida que o nosso século se aproxima do seu término e que nos aproximamos de um novo milênio, a sobrevivência da humanidade dependerá de nossa alfabetização ecológica, de nossa capacidade para entender esses princípios da ecologia e viver em conformidade com eles" (Capra, 1996). Assim mesmo, a tentativa obstinada de nosso grupo para compreender biodiversidade e suas "questões subjacentes" já foi considerada "apenas um sonho" por alguns, ao passo que outros ponderam que os objetivos da fitoquímica em países em desenvolvimento deveriam ser restritos à busca de princípios bioativos (discussão anexa a Gottlieb \& Borin, 1994b). Opiniões desse tipo fazem com que a causa da biodiversidade tropical continue sendo um dos maiores problemas teóricos da biologia evolutiva. Eis por fim uma questão autenticamente nacional que podemos e devemos abranger em todas as suas facetas, desde as micromoleculares às macroambientais.

Tão vastos são os horizontes de investigação em biodiversidade que requerem cooperação de considerável número de especialistas das mais diversas áreas. Em exemplo convincente, vinte investigadores perfazem o grupo norte-americano Faunmap, financiado pela National Science Foundation (Graham et al., 1996). Claro que uma estratégia interdisciplinar única deve ser proposta e coordenada por órgãos oficiais de fomento ou por institutos oficiais de pesquisa também no Brasil. Somente dessa maneira, apesar das projeções alarmantes de depauperamento da riqueza natural, será possível chegarmos a uma coexistência mutualista ou, pelo menos, mais inteligente com a natureza.

\section{Agradecimentos}

Os autores agradecem o apoio recebido do Conselho Nacional de Desenvolvimento Científico e Tecnológico, da Academia Brasileira de Ciências e da Fundação de Amparo à Pesquisa do Estado do Rio de Janeiro. 


\section{Referências}

Beardsley T 1994. Green Economics. Scientific American 271(1): 86.

Borin MR de MB \& Gottlieb OR 1993. Steroids, Taxonomic Markers? Pl. Syst. Evol. 184: 41-76.

Borin MR de MB \& Gottlieb OR 1997. Natural Products and Neotropical Biodiversity, p. 95-103. In P Naranjo \& A Crespo (Eds.) - Etnomedicina. Progressos Italo-Latinoamericanos, v.II. Memórias del IV Congresso Italo-Latinoamericano de Etnomedicina "Felice Fontana”. Ediciones Abya-Yala, Quito, Ecuador.

Bosisio BM, Borin MR de MB \& Gottlieb OR 1998. Quantitative Chemical Biology. I. Biological Mapping of Lauraceae. Anais da Academia Brasileira de Ciências, no prelo.

Buckingham J 1994-1997. Dictionary of Natural Products, v.1-10. Chapman and Hall, London.

Capra F 1996. A Teia da Vida. Uma Nova Compreensão Científica dos Sistemas Vivos. Cultrix, São Paulo. 235 pp.

Eldredge N 1995. Reinventing Darwin. The Great Debate at the High Table of Evolutionary Theory. John Wiley \& Sons, Inc., New York.

Gottlieb OR 1982. Micromolecular Evolution, Systematics and Ecology. An Essay into a Novel Botanical Discipline. Springer Verlag, Berlin.

Gottlieb OR 1990. Phytochemicals: Differentiation and Function. Phytochemistry 29: 1715-1724.

Gottlieb OR \& Borin MR de MB 1994a. The Diversity of Plants: Where Is It? Why Is It There? What Will It Become? Anais da Academia Brasileira de Ciência 66(Suplemento): 55-83.

Gottlieb OR \& Borin MR de MB 1994b. Biodiversity as Base for Angiosperm Prospection, p. 23-36. In PR Seidl (Ed.). Proceedings of Workshop: The use of Biodiversity for Sustainable Development: Investigation of Bioactive Products and their Commercial Applications. Associação Brasileira de Química, Rio de Janeiro.

Gottlieb OR \& Borin MR de MB 1996. Morphological and Metabolic Differentiation of Rubiaceae by Ecogeographical Transitions in South America. Opera Bot. Belg., 7: 213-230.

Gottlieb OR \& Borin MR de MB 1997a. Shamanism versus Science in the Search for Useful Natural Products, p.123-135. In L Verotta (Ed.) - Virtual Activity, Real Pharmacology - Different Approaches to the Search for Bioactive Natural Compounds. Research Signpost, Trivandrum.

Gottlieb OR \& Borin MR de MB 1997b. Natural Products Research in Brazil. Ciência \& Cultura 49 (5/6): 315-320.

Gottlieb OR \& Borin MR de MB 1997c. Chemosystematical Clues for the Search of Nutraceuticals, p. 201207. In P Naranjo \& A Crespo (Eds.) - Etnomedicina. Progressos Italo-Latinoamericanos, v. II. Memórias del IV Congresso Italo-Latinoamericano de Etnomedicina "Felice Fontana". Ed. Abya-Yala, Quito, Ecuador.

Gottlieb OR \& Borin MR de MB 1998a. Quantitative Chemical Biology. IV. Analogies of Metabolical Mechanism and Biological Evolution. Anais da Academia Brasileira de Ciências, no prelo.
Gottlieb OR \& Borin MR de MB 1998b. The Neotropical Plant Web. In M de LD Freitas (Ed.) - Projeto Céu do Novo Mundo. no prelo.

Gottlieb OR \& Borin MR de MB 1998c. Natural Products and Evolutionary Ecology. Pure and Applied Chemistry, no prelo.

Gottlieb OR \& Borin MR de MB 1998d. Evolution of Angiosperms via Modulation of Antagonisms. Phytochemistry, no prelo.

Gottlieb OR \& Borin MR de MB 1998e. A Universalidade de Antagonismos. O potencial direcionador da ação do tempo modulado pelo potencial criativo da adaptação ao espaço. Ed. UFRJ, Rio de Janeiro, no prelo.

Gottlieb OR, Borin MR de MB \& Bosisio BM 1995a. Lignification: An Evolutionary Perspective. Anais da Academia Brasileira de Ciência 67 (Suplemento): 355-361.

Gottlieb OR, Borin MR de MB \& Bosisio BM 1995c. Chemosystematic Clues for the Choice of Medicines and Food Plants in Amazonia. Biotropica 27: 401406.

Gottlieb OR, Borin MR de MB \& Kaplan MAC 1995b. Biosynthetic Interdependence of Lignins and Secondary Metabolites in Angiosperms. Phytochemistry 40(1): 99-113.

Gottlieb OR, Kaplan MAC \& Borin MR de MB 1996a. Biodiversidade. Um Enfoque Químico-Biológico. Ed. UFRJ, Rio de Janeiro, 267 pp.

Gottlieb OR, Borin MR de MB \& Bosisio BM 1996b. Trends of Plant Use by Humans and Nonhuman Primates in Amazonia. American Journal of Primatology 40: 189-195.

Graham RW, Lundelius Jr. EL, Graham MA, Schroeder EK, Toomey Iii RS, Anderson E, Barnosky AD, Burns JA, Churcher CS, Grayson DK, Guthrie RD, Harington CR, Jefferson GT, Martin LD, McDonald HG, Morlan RE, Semken Jr. HA, Webb SD, Werdelin L, Wilson MC 1996b. Spatial Response of Mammals to Late Quaternary Environmental Fluctuations. Science 272: 1601-1606.

Kornberg A 1987. The Two Cultures: Chemistry and Biology. Biochemistry 26: 6888-6891.

Mayr E 1991. One Long Argument. Charles Darwin and the Genesis of Modern Evolutionary Thought. Harvard University Press, Cambridge, $186 \mathrm{pp}$.

Pagotto CLA da C, Barros JRT, Borin MR de MB \& Gottlieb OR 1998. Quantitative Chemical Biology. II. Chemical Mapping of Lauraceae. Anais da Academia Brasileira de Ciência, no prelo.

Sagan C 1996. O Mundo Assombrado pelos Demônios. A Ciência Vista como uma Vela no Escuro. Companhia das Letras, São Paulo. 22 pp.

Sporne KR 1980. A Re-Investigation of Character Correlations Among Dicotyledons. New Phytology 85: 419-499.

Wilson EO 1992. The Diversity of Life. Belknap Press of Harvard University Press, Boston. 424 pp.

Zocher DHT, Borin MR de MB \& Gottlieb OR 1998. Quantitative Chemical Biology. III. Chemical Mapping of Dilleniidae. Anais da Academia Brasileira de Ciência, no prelo. 